anthropology \& materialism

\section{Anthropology \& Materialism}

A Journal of Social Research

$4 \mid 2019$

Art and Technique: A Framework of Unaccomplished Promises

\title{
Den Algorithmus unterwandern
}

Digitale Maskeraden als aktivistisch motivierte Kunstform der Gegenwart

Infiltrate the Algorithms. Digital Masking as an Artistic Form for Our Present Infiltrer les algorithmes. Le masquage numérique, une forme artistique d'activisme pour notre présent Infiltrar los algoritmos. La máscara digital, una forma artística de activismo resistente para nuestro presente

Jana Johanna Haeckel

\section{(2) OpenEdition}

Journals

Electronic version

URL: http://journals.openedition.org/am/1112

DOI: $10.4000 / \mathrm{am} .1112$

ISSN: 2364-0480

Publisher:

CETCOPRA, CRASSH - Center for Research in the Arts Social Sciences and Humanities, Fakultät

Gestaltung - Universität der Künste Berlin

Electronic reference

Jana Johanna Haeckel, « Den Algorithmus unterwandern », Anthropology \& Materialism [Online], 4 |

2019, Online erschienen am: 21 November 2019, abgerufen am 23 November 2019. URL : http:// journals.openedition.org/am/1112 ; DOI : 10.4000/am.1112

This text was automatically generated on 23 novembre 2019 .

Tous droits réservés 


\section{Den Algorithmus unterwandern}

Digitale Maskeraden als aktivistisch motivierte Kunstform der Gegenwart

Infiltrate the Algorithms. Digital Masking as an Artistic Form for Our Present Infiltrer les algorithmes. Le masquage numérique, une forme artistique d'activisme pour notre présent

Infiltrar los algoritmos. La máscara digital, una forma artística de activismo resistente para nuestro presente

Jana Johanna Haeckel

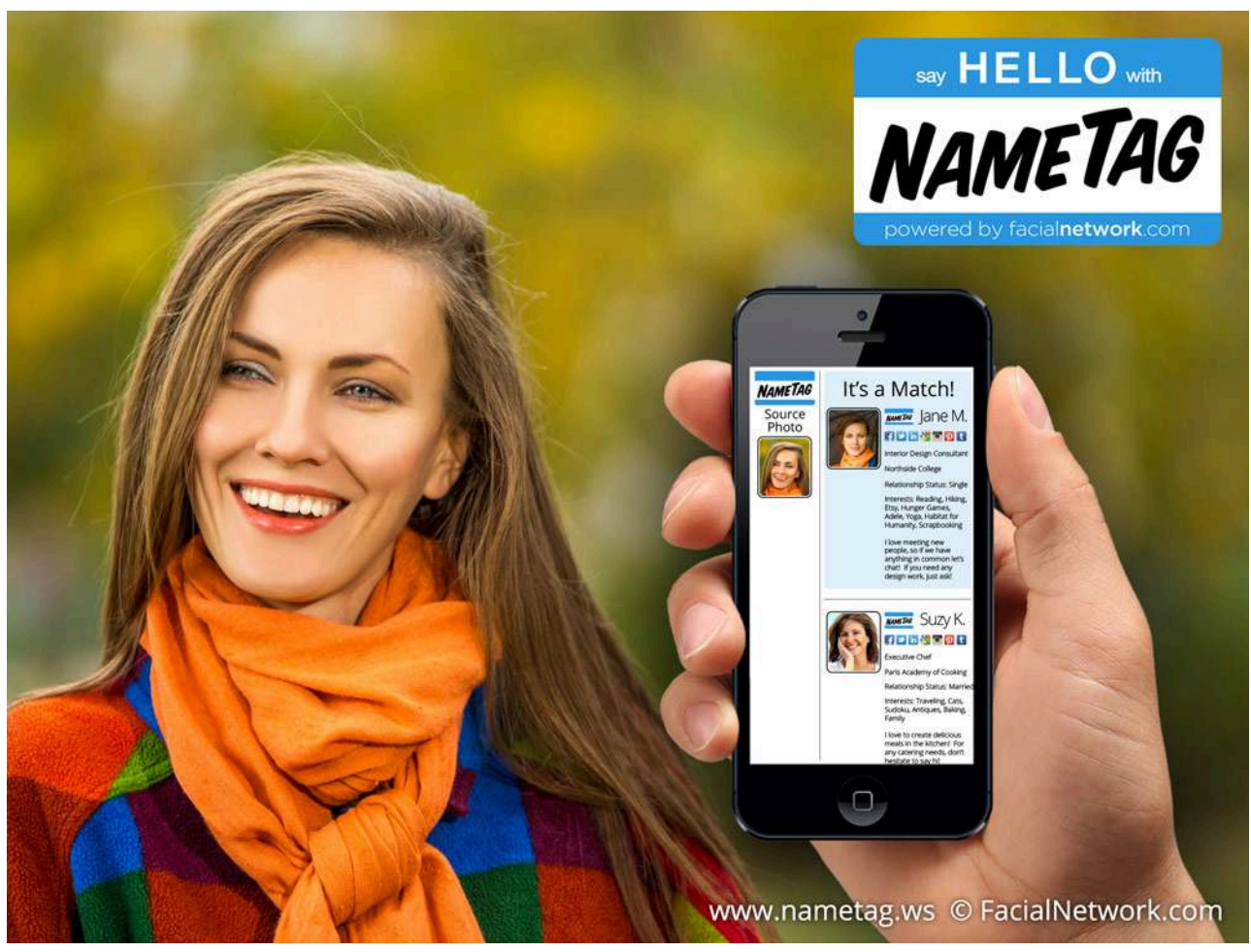

Werbekampagne für die Gesichtserkennungs-App Nametag 


\section{Einführung}

1 Egal ob auf digitalen Netzwerken wie Instagram oder facebook, als Gesichts- und Körperscan in der Sicherheitskontrolle oder als Vorschlag einer "facialen Wende“ in den Wissenschaften (Weiss 2012: 30). Gesichter sind als Bedeutungsträger allgegenwä rtig. Bedenklicherweise wird die individuelle Einzigartigkeit des Gesichts, seine Identität, jedoch immer öfter als Anwendungstool digitaler Software-Programme missbraucht, welche seine visuelle Oberfläche zu Markt- und Überwachungszwecken zu instrumentalisieren wissen. Während traditionelle Darstellungen des Gesichts noch auf der Vorstellung einer individualisierten Innenansicht des Menschen basierten und einen psychischen oder auch "seelischen" Ausdruck einzufangen versuchtenwird das digitale Gesichtsbild der Gegenwart zur entleerten Schablone der Überwachungsgesellschaft. App-Anwendungen wie FacialNetwork, NameTag oder Google Glass deuten das Gesicht als "Inter-Face" und reduzieren seine psychologische und physiognomische Unverwechselbarkeit auf eine kleinste mathematische Information im Sinne der facial recognition (Chayka 2014; Bainbridge, Khosla, Torralba, Oliva 2013. Zur jüngsten Gesichtserkennungsapp FindFace aus Russland: Walker 2016 sowie zur flächendeckenden facial recognition in China: Paul Mozur 2018). Somit wird das Gesicht - oder vielmehr sein Abbild - im digitalen Zeitalter zum Symptom einer Krisenhaftigkeit. Drei Hauptfaktoren wirken dabei auf die veränderten Erscheinungsund Repräsentationsformen des menschlichen Gesichts und Körpers ein: (1.) Das Zeitalter des Digitalen und sein veränderter Umgang mit dem Wahrheitsgehalt von massenmedial distribuierten Bildern sowie das aus Algorithmen bestehende Bildprodukt, das viele nicht mehr nachweisbare Veränderungen durchläuft; (2) neue Technologien wie Facial App-Anwendungen, die eine Kontroll-basierte Abbildung und Vorstellung von Wirklichkeit hervorbringen; (3) der 11. September und seine Auswirkungen auf eine neue Überwachungs-, Bild- und Körperpolitik, d.h. die politische und ökonomische Vereinnahmung sogenannter „Gesichts- und Körperoberflächen“.

2 Mein Essay nimmt die These einer "facialen Wende" im digitalen Zeitalter als Ausgangspunkt und versucht am Beispiel exemplarischer künstlerischer Werke, die sich mit neuesten biometrischen Technologien und facialen Überwachungsmethoden befassen, einen Erkenntnisgewinn zu erlangen. Zentral ist dabei für mich die Frage, wie es mittels künstlerischer Reflexion und visuellen Handelns gelingen kann, einen verantwortungsvollen, kritischeren Umgang mit dem vielschichtigen Thema medialer Darstellung von Gesicht und Körper zu erzielen. Welche Art von Gesichts- und Körperbildern bringen die neuen digitalen Technologien hervor? Und wie ändern sie das Verhältnis von Sichtbarkeit/Unsichtbarkeit des digital erfassten Körpers?

Die für meine Analyse ausgewählten Arbeiten bilden einen exemplarischen Ausschnitt von Gesichtsmaskierungen in der zeitgenössischen Bildpraxis ab, die auf visuelle Kontrolle und algorithmisch gelenkte Blickregime reagieren. Vor dem Hintergrund des Zustands einer „liquid surveillance“(Bauman \& Lyon 2013) problematisieren sie das neue Gesichts- und Körperbild der digitalen Gegenwart und fragen danach, wie der 
verloren gegangene, ehemals autonome Körper dahinter adressiert sowie reanimiert werden kann.

\title{
I. Sichtbarkeit vs. Kontrolle - Gesicht und Bild in der Krise
}

\author{
Jedes Gesicht verkörpert eine Paradoxie: Ein \\ Anwesendes, das fehlt; ein Abwesendes, das \\ vortritt und bindet. Gesichter sind überall und \\ nirgends, nah und ganz fern, vertraut und völlig \\ fremd: Kraftfelder der Anerkennung - und \\ Masken des Todes. Jede ernsthafte Physiognomik \\ (sei es in der Philosophie oder in der Kunst) \\ beginnt daher mit einer Kritik des Gesichts, mit \\ einer behutsamen Öffnung des kryptischen - \\ niegeschauten - Zeichens am eigenen Leib. \\ Thomas Macho, „Gesichtsverluste“,1996, 25
}

Bereits 1996 diagnostizierte der Kulturwissenschaftler Thomas Macho im Zuge der fortschreitenden Digitalisierung, „wir leben in einer facialen Gesellschaft, die ununterbrochen Gesichter produziert.“(Macho 1996: 26; Macho 2011). Macho bezieht seine Aussage auf eine veränderte Bildkultur, die im Zuge des technischen Wandels und poststrukturalistischen Identitätskrisen an dem Individualitäts- und Authentizitätsversprechen des menschlichen Gesichts rüttelt: Die Massenmedien brachten eine visuelle Überschwemmung an medial abgebildeten Gesichtern, deren Singularität und Wirkkraft durch neue Bildbearbeitungsprogramme wie Photoshop oder Morphing entwertet wurde. Eine der ersten künstlerischen Reaktionen auf das sogenannte "digital trouble“ waren die fotografischen Kompositbilder Nancy Bursons, die das Ergebnis von Überlagerungen verschiedener ikonischer Porträts sind (u.a. Mankind und Bigbrother, 1983). Desweiteren können Inez van Lamsweerdes beunruhigende Porträtserien deformierter und hybridisierter Frauenkörper (Sasja 90 60 - 90, 1992) und Aziz\&Couchers retouschierte Porträts genannt werden, auf denen entmenschlichte Wesen ohne Augen und Münder den Bezug zur sinnlichen Welt verloren haben (Dystopia, 1994-95). Die künstlerischen Arbeiten visualisierten das Unbehagen, das die veränderte digitale Bildbearbeitung sowie Biopolitik auslöst, indem sie traditionelle Vorstellungen von Ähnlichkeit und Identität des Subjekts dekonstruieren. Laut Hubertus von Amelunxen zeigen sie, dass „Identität in der Auflösung begriffen“(Amelunxen 1995: 122) ist und entwickeln entpersonifizierte Bilder von Typen, Masken oder auch „Projektionen möglicher Antlitze“, die das Individuum hinter sich gelassen haben (Hüsch 2007). Hierfür konzentrieren sich die Künstler/-innen der 1990er Jahre bei ihren Gesichtsdarstellungen vornehmlich auf das (post-)fotografische Medium (Mitchell 1992). 


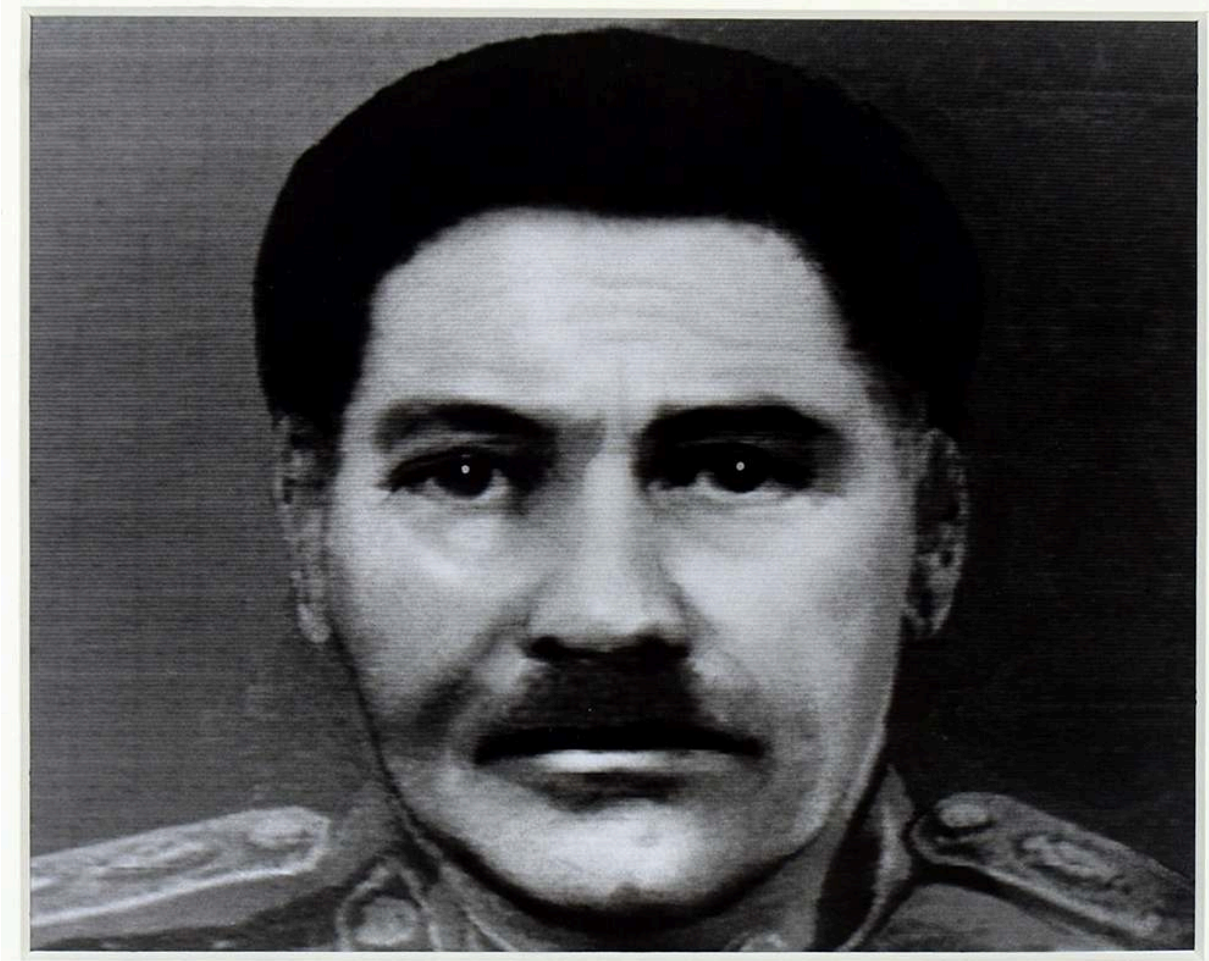

Nancy Burson, Big Brother, Kompositbild, 1983

5 Im Prozess der Digitalisierung entstehen Gesichter, die zunehmend maskenhaft und überindividuell sind; sie besitzen keinen authentischen Kern oder tiefenpsychologische Bedeutung, die es zu entschlüsseln gelte. Dem Kunsthistoriker Hans Belting zufolge wird das Gesicht in der digitalen Gesellschaft zum „Interface“, zur reinen „Oberfläche“, auf der „die Gesichter, isoliert von jeder Körperlichkeit, kommen und gehen“(Belting 2013, 230). Belting vertritt die These, dass das Gesicht bis heute eines der sinnstiftendsten Elemente der Kulturgeschichte darstellt und sich deshalb über die Grenzen der Kunstgeschichte hinaus als Erkenntnismittel der Gegenwartsanalyse eigne. Er kritisiert die zunehmende „Entkörperlichung“ unserer digitalen Gegenwart und fragt nach den realen Körpern hinter den Bildern, die durch die "Kulturrevolution" elektronischer Medien mit neuen Herausforderungen und Infragestellungen zu kämpfen haben (Flusser 1992:11). Während für den Philosophen Emmanuel Lévinas im Dialog von Angesicht zu Angesicht die Grundlage einer ethischen und demokratisch intakten Gesellschaft lag, ersetzt in der digitalisierten Gegenwart das Spiel mit Gesichts-Oberflächen bzw. Avatars die vis-à-vis Begegnung (Keenan, 2013). Der Bildwissenschaftler Oliver Grau beobachtet diesbezüglich, dass die „Interfacegestaltung" in der Medienkunst zunehmend zum "Politikum" werde, wobei ein Streit um "natürliche" vs. "kritische" Interfaces entbrannt sei, die ihre "medialen und ikonischen Grundlagen verschleiern oder ins Bewusstsein heben" (Grau 2001). Er bezweifelt, dass die psychologisch-manipulativen, interaktiv erfahrenen Illusionsräume und virtuellen Interaktionen eine distanzierte Rezeption zulassen, da erst die "Distanzgeste des Rezipienten" eine kritische Haltung ermögliche, welche wichtiger "Teil des zivilisatorischen Prozesses" sei (Grau 2001: 183). Grau konstatiert, dass je „körperintimer" die Interface-Gestaltung werde, desto intensiver werde auch die „illusionäre Entgrenzung mit dem Datenraum“ (Grau 1997). Als Konsequenz fordert er für die Psychologie, Informatik und insbesondere die Kunstgeschichte eine „, neue 
Schule des Bildes, die das Rüstzeug für den Umgang mit den lebendigen Bildern vermittelt" , um mit den neuen Herausforderungen umgehen zu können (Grau 1997).

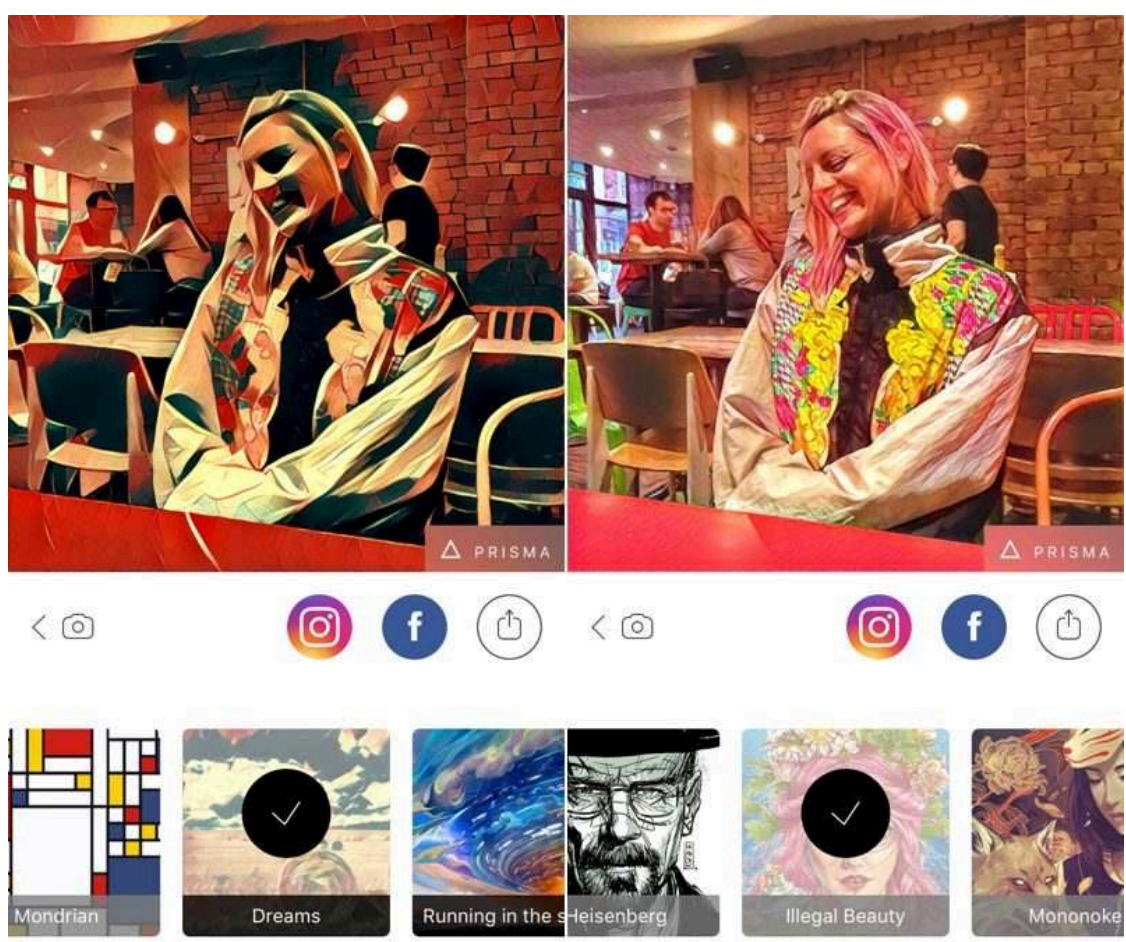

Screen-Shot der Prisma-App Anwendung

Diese neue Bildschule scheint vor allem für den alltäglichen Gebrauch von AppAnwendungen nötig. Mobile Smartphone-Apps, deren Gebrauch im Alltag nahezu untrennbar mit unseren Körpern verbunden ist, konfrontieren die Zivilgesellschaft mit einer neuen Form subtil gesteuerter Vermarktung und Überwachung, deren Mittel und Zweck den Benutzern oft unbekannt bleiben: So kann etwa ein mit der kostenloserwerbbaren App-Anwendung Prisma aufgenommenes Smartphone-Porträtbild innerhalb weniger Sekunden in ein "Kunstwerk" nach den kunsthistorischen Vorbildern Van Gogh, Mondrian oder Picasso verwandelt werden. Im "Tausch" gegen den Schnappschuss überlässt der Nutzer der „Prisma“-App laut Datenschutzerklärung ${ }^{1}$ die Bild- und Nutzungsrechte - ähnlich wie bei facebook oder Instagram - dem russischen Prisma-Konzern, der das Bild, sobald es auf den Server zur Bearbeitung hochgeladen ist, weiterverwenden darf. Das gleiche gilt für die Log- und Ortsdaten sowie weitere vertrauliche Informationen der Nutzer. Dies zeigt, dass im Zeitalter digitaler Technologien nicht nur die Definition des Porträts neu überdacht, sondern auch die Frage nach Bild- und Persönlichkeitsrechten kritisch gestellt werden muss. Die attestierte oberflächliche "Gesichthaftigkeit" zirkulierender Bilder im digitalen Zeitalter ermöglicht eine immer perfektioniertere Kontrollier- und Manipulierbarkeit algorithmisch definierter Gesichtsoberflächen. Im Gegensatz zur zwischenmenschlichen Gesichtserkennung, bei der die Gegenüber durch komplexe psychologisch-perzeptive Merkmale wie Mimik, Emotion etc. erkannt werden, folgt die technische Variante einem reduktiven biometrischen Verfahren. Sie basiert auf einem kriminalistischen und forensischen Interesse, mit dem Ziel, verdächtige Personen zu identifizieren und $\mathrm{zu}$ authentifizieren. Was genau sind die Hintergründe dieser fragwürdigen technischen Entwicklung und wie gestalten sich die systemischen 
Zusammenhänge in Hinblick auf die facial recognition-Technologie und deren Entwicklung?

\section{Liquid Surveillance}

7 Die neuen technischen Möglichkeiten der massenhaften und seriellen Reproduktion erleichterten die staatliche Erfassung, etwa durch das fotografische Passbild. Hierdurch wurde es möglich, physiognomische Individualität zugunsten von Normierung umzudeuten und zu instrumentalisieren. Fotografierte Gesichter werden infolge dessen nicht mehr nur im Sinne der Studiofotografie als persönliche Erinnerungsbilder produziert, sondern zunehmend Teil staatlicher Polizei- und Registrierungsarchive, welche das individuelle Antlitz mit einer Schablone gleichsetzen und zwecks bürokratischer bzw. kriminologischer Erfassung sammeln (Sekula 2003; Belting 2013: 234-249): eine Praxis, die sich bis in die Gegenwart gehalten hat und die mit Einzug digitaler Techniken weiter perfektioniert wurde. ${ }^{2}$ Softwareentwicklungen wie NameTag oder Facialnetwork stellen ihre virtuellen Bildarchive von Gesichtsaufnahmen u.a. der NSA und dem FBI zur Verfügung, die im Zuge einer veränderten Sicherheitspolitik nach dem 11. September Überwachungs- und Kontrollmethoden professionalisieren. Im öffentlichen Raum installierte Videokameras nehmen ununterbrochen - und ohne Wissen der Betroffenen - Bilder- bzw. Gesichtsdaten auf, die mithilfe von Gesichtserkennungsalgorithmen jederzeit ausgewertet werden können. Es wird möglich die „Bewegungsmuster aller Menschen nachzuzeichnen, deren biometrische Daten bereits über Fahndungsfotos oder andere Formen der Registrierung in ein System miteinander vernetzter Datenbanken erfasst wurde“ (Waligorski 2016). Dabei gelingt es durch die automatisierte Lokalisierung ("facial detection"), Personen anhand ihrer spezifischen Gesichtsgeometrie zu identifizieren. Hieraus folgt: je omnipräsenter das Gesicht ist, umso mehr unterliegt es auch der staatlichen Kontrolle und Manipulation.

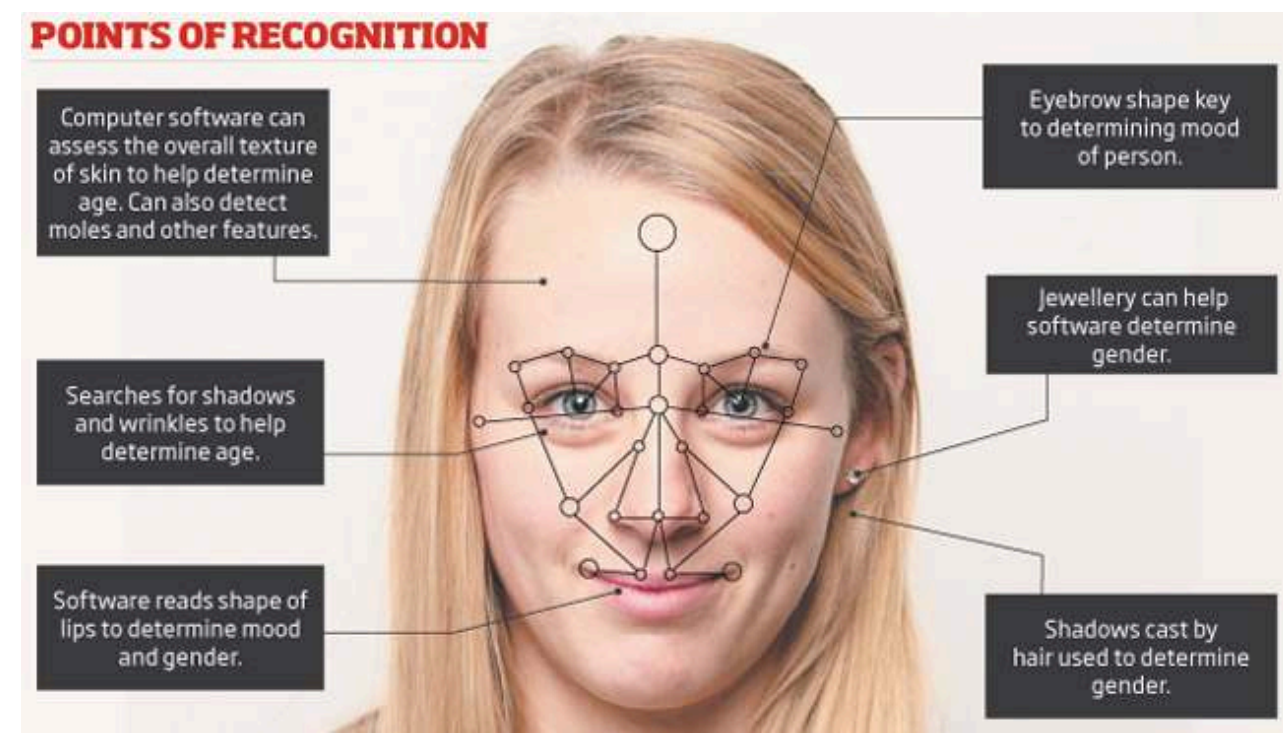

Beispiel der Analysekriterien von Gesichtserkennungssoftware, die für CCTV Kameras im öffentlichen Raum angewendet wird

8 Seit den Terroranschlägen vom 11. September 2001 wird die automatisierte Gesichtserkennung zunehmend als Prävention zur Ortung sogenannter "faces of 
terror" propagiert. Die Soziologen Zygmunt Bauman und David Lyon deuten die permanente Überwachung als Signum der Moderne und denken den von Bauman entwickelten Begriff der „flüchtigen Moderne“, der die exterritorialen und mobil gewordenen Machtstrukturen der modernen Gesellschaft sowie deren unübersichtige und flüchtige Hierarchien beschreibt (Bauman 2003), in Hinblick auf Überwachung und Kontrolle weiter: In Folge der Anschläge des 11. September 2001 seien die staatlichen Überwachungsmethoden in einen "flüssigen bzw. gasförmigen Zustand" übergegangen und vermögen es in Lebensbereiche einzudringen, die früher nur am Rande oder gar nicht überwacht wurden:

Surveillance is a key dimension of the modern world [...]. Not only in London and New York but also in New Delhi, Shanghai and Rio de Janeiro video cameras are a familiar sight in public places. Travellers through airports everywhere are conscious that they not only have to negotiate twentieth-century passport control but also newer devices such as body scanners and biometric checks that have proliferated since 9/11. And if these have to do with security, other kinds of surveillance, relating to routine and mundane purchases or online access or participation in social media, are also increasingly ubiquitous. We have to show ID, insert passwords and use coded controls in numerous contexts from making online purchases to entering buildings. Every day, Google notes our searches, prompting customized marketing strategies (Bauman \& Lyon 2013, 8).

Das System der "liquid surveillance" funktioniere vor allem über verschleierte, algorithmisch basierte Überwachungssysteme und beruhe vornehmlich auf missbräuchlichem Umgang mit Daten, welche die Nutzer den Megakonzernen wie Google oder facebook meist unwissentlich zur Verfügung stellen. Im Zuge dessen wurden zahlreiche Gesichtserkennungssoftwares entwickelt, die von Geheimdiensten und Privatkonzernen eingesetzt werden, um Bilddatenbanken nach bestimmten Gesichtsmerkmalen zu durchsuchen. Vermittels automatisierter Lokalisierung ("facial detection") wird es möglich, Personen anhand ihrer spezifischen Gesichtsgeometrie und Textureigenschaften des Kopfes in Frontalansicht zu identifizieren. So entwickeln etwa Google und Facebook immer komplexere Programme, die lernfähig sind und sich an den unzähligen Bilddatenbanken abarbeiten, um ein immer detaillierteres und umfassenderes Bildarchiv menschlicher Gesichter aufzubauen. Facebook verfügt beispielsweise nicht nur über die Namen der online "Freunde", sondern kann mittels der entwickelten Software auch alle Menschen identifizieren, die auf den geposteten Fotografien angezeigt werden. Zusätzlich ist es durch die Nutzer-bedingten Angaben zu Studium, Job, Reise etc. möglich, Hintergrundinformationen zu den Fotografien herauszufinden. Mit den ins System eingespeisten Informationen über Alter, Geschlecht, sexuelle Orientierung etc. wird schrittweise ein "ultimatives Gesichtserkennungsnetzwerk" aufgebaut, wie Morozov kritisch anmerkt (Morozov 2011). Dieses Gesichtserkennungsnetzwerk setzt sich vielfachen Bildinstanzen/ Bildquellen zusammen. Gleichzeitig liefern Videokameras in öffentlichen Räumen kontinuierlich Bilddaten, die mithilfe von Gesichtserkennungsalgorithmen und ohne die explizite Einwilligung der Betroffenen ausgewertet werden können. Hierdurch wird es möglich, die Bewegungsmuster aller Menschen im öffentlichen und im privaten Raum nachzuzeichnen. Besonders bereits erfasste Menschen, deren biometrische Daten bereits in ein System miteinander vernetzter Datenbanken eingepflegt wurden, bilden Verknüpfungen in diesem ultimativen Bildarchiv und tragen zu seiner Komplettierung bei. Die neue digitale Überwachungstechnologie gestaltet sich als Potenzierung optisch-elektronischer Videoüberwachungssysteme (Closed-Circuit Television oder 
auch CCTVs), welche seit den 1960er Jahren zur Überwachung in den öffentlichen Raum eingeführt wurden. ${ }^{3}$ Der Politologe und Vordenker des digitalen Wandels, Evgeny Morozov, warnt deshalb vehement vor der Bedrohung, die der Einsatz staatlicher biometrischer Gesichtserkennungssoftware für die Demokratie habe (Morozov 2011). Mit Blick auf die Occupy-Demonstrationen in den USA geschieht die missbräuchliche Analyse der Gesichts-Daten beispielsweise auch, wenn auf Youtube oder facebook hochgeladene Videos von Demonstranten von der Polizei ausgewertet und für Ermittlungszwecke eingesetzt werden. Die Kriegs- und Sicherheitspolitik nach 9/11 sei dabei lediglich ein Vorwand, um das biometrische Überwachungssystem auszubauen, mit dem Ziel eines flächendeckenden Einsatzgebiets (Morozov 2011). Wie kann dem Missbrauch von persönlichen Daten entgegen gewirkt werden und welche Formen des Protests gibt es, die vom virtuellen auf den realen (Rechts-)Raum übergreifen? Wie kann dem Gesicht eine neue Autonomie zugesprochen werden, mehr noch: Wie kann es wiederbelebt werden?

\section{Visual Actionism - Gesichtsentzug und - zerstörung als Protestform}

Bezeichnenderweise lassen sich in jüngster Zeit auffallend viele visuelle Strategien in der zeitgenössischen Kunst und visuellen Kultur erkennen, die einen kritischen oder auch subversiven Gegenentwurf zu der Verfallsthese der "Oberflächlichkeit“ (Macho) massenhaft zirkulierender, entleerter und kontrollierter (Medien-)Gesichter präsentieren. Sie widmen sich der Aufgabe, „den industriell verfügbaren Bildmedien in wörtlichem Sinne die Masken vom Gesicht zu reissen“ und Stereotype zu überwinden (Belting 2013: 272). Ein viel verbreitetes Bildgenre stellt diesbezüglich der Gesichtsentzug dar: auf den Darstellungen wird das Gesicht entweder verborgen, ist abgewendet oder wird zerstört, wie etwa im Guerilla-Phänomen des „Defacings“, das eine lange Tradition verfolgt. Unter Defacing versteht man das Übermalen und Zerstören von öffentlichen Gesichtern auf Plakaten, Leinwänden und Monumenten, oftmals mit dem Ziel, einen Machtwechsel zu proklamieren. Solche ikonoklastischen Akte richteten sich ehemals gegen Kultbilder der Kirche oder Standbilder/Monumente von Oberhäuptern, deren Herrschaft symbolisch durch die Präsenz im Bild ausgedrückt wurde. Als zeitgenössisches Beispiel kann u.a. die Zerstörung von Wandplakaten mit Mubaraks Gesicht durch Demonstranten während der ägyptischen Revolution im Januar 2011 genannt werden - eine technisch zwar noch im Analogen verhaftete, aber durchaus auf digitale Phänomene übertragbare visuelle Gegenstrategie (Belting 2013: 222; De Raedt 2015). Weitere Defacing-Proteste afro-amerikanischer Bürgerrechtler fanden u.a. in Harvard statt, wo Studenten die Gesichter ehemaliger Professoren überklebten, um auf die Chancenungleichheit der universitären Lehre aufmerksam zu machen (Bidgood 2015) oder im Rahmen der partiellen Zerstörung einer Robert E. LeeeStatue an der Duke University in North Carolina (Simpson 2017). Die Defacing-Aktionen können als politisch motivierte Protestform bezeichnet werden, welche die Macht des Gesichts/Bildes als Teil visueller Propaganda voraussetzt. Das entindividualisierte, repräsentative Herrscherbild wird aus der Oberfläche zirkulierender Bilder herausgelöst und beschmutzt bzw. zerstört, um auf dahinterliegende politische/ historische Konflikte hinzuweisen. Es wird in einem ikonoklastischen "wiederbelebt" und gleichzeitig entlarvt. 
11 Als weitere Form des Gesichtsentzugs lässt sich ein verstärktes Auftreten kollektiver Gesichtsmasken im öffentlichen Raum beobachten, etwa bei aktivistischen Bewegungen wie Anonymous (die Guy Fawkes-Maske), dem Schwarzen Block oder Pussy Riot. Auch in der Pop Kultur (Daft Punk, Gangster Rapper Sido, Cro u.a.) (Leber, Rink 2012) sowie in der Mode (Maison Margiela, Gucci u.a.) (Lynch, Strauss 2007) tauchen immer mehr Maskierungsformen auf, welche als subversive Gegenstrategie zur gesellschaftlichen Gesichtskontrolle und Normierung gedeutet werden können. Auch in der Bildenden Kunst ist das Phänomen des Gesichtsentzugs bzw. der Maskierung vorzufinden, etwa in Signe Pierces feministisch motivierter Performance American Reflexxx. ${ }^{4}$ Wie Alexandra Waligorski anmerkt, werden bestimmte Formen der Gesichtsmaskierungen in den Künsten seit einiger Zeit vermehrt als Strategie der "Countersurveillance" eingesetzt, welche "die Mechanismen der Gesichtserkennung und -normierung abwehrt und gleichzeitig sichtbar macht" (Waligorski 2016). Interessanterweise formulieren die Masken-Arbeiten eine Kritik an neusten Formen biometrischer Gesichtskontrolle, die das seit dem 19. Jahrhundert existierende Prinzip fotografischer Gesichts- und Körpererfassung zur kriminologischen Erfassung in digitale Formate umgewandelt und perfektioniert haben. Mit Blick auf das Gesicht stellt sich diesbezüglich die Frage, inwieweit die Praxis der Maskerade den Betrachtern zwar die vis-à-vis Begegnung verwehrt, diese im Gegenzug dafür jedoch ihre Autonomie und letztlich auch ihre Individualität zurückgewinnen, da sie unerkannt bzw. unlesbar bleiben. So verweist die Strategie des Gesichtsentzuges nicht zuletzt auch auf eine Methode, den herrschenden Machtsystemen zu entkommen, wie es bereits von Deleuze/Guattari im Zuge ihrer berühmten Rationalismus- und Kapitalismus-Kritik in Milles Plateaux formuliert wurde: “das Gesicht muss zerstört werden" (Deleuze, Guattari 1992). Die radikale Aussage der Gesichtstheorie von Deleuze/Guattari lautet: Es gibt kein “Jenseits der Maske", keinen "authentischen Kern", den es zu entdecken gilt (Deleuze, Guattari 1992: 476). Die humanistische Vorstellung einer sich physiognomisch und psychologisch manifestierenden Innerlichkeit, einer "Authentizität", die sich im Gesicht widerspiegelt und die man zu entdecken gewillt ist, wird demnach negiert. Nach Deleuze/Guattari geht es vielmehr darum, die Gesichts-Maske spielerisch zu verwenden und dadurch zu versuchen, den kontrollierenden Blickregimen $\mathrm{zu}$ entkommen. Welches subversive Potential liegt im Gesichtsentzug?

\section{Liquid Identities - Das Gesicht als virtuelle Maske bei Zach Blas, Sterling Crispin und Martin Backes}

12 Der Künstler und Wissenschaftler Zach Blas (*1981, USA) entwickelt seit 2011 seine fortlaufende Facial Weaponization Suite-Reihe, welche Fragen biometrischer Gesichtserkennung aufgreift und deren selektive, reduktionistische Vorgehensweise visualisiert. Die Serie besteht aus insgesamt vier Masken-Typen, die in speziellen Workshops entwickelt werden und auf den biometrischen Gesichtsinformationen der Teilnehmer aufbauen. Im Mittelpunkt steht die Auswertung der Gesichtsinformation spezifisch ausgewählter gesellschaftlicher Randgruppen, in diesem Fall queere, lesbische, mexikanische und schwarze Minderheiten der westlichen Gesellschaft (Blas 2015: 85-86). Jede Randgruppe bekommt einen bestimmten Maskentypus zugeteilt - die queere Minderheit beispielsweise die Fag Face Mask (2012), deren pinke blasenartige Oberfläche auf einem 3D-Print spezifischer, vermengter Datensätzen von queeren 
Männern basiert. Zusätzlich zu der in offenen Workshops entwickelten kollektiven Maske, die sich aus den Gesichtsdaten aller Teilnehmer speist, werden in der Gruppe aktuelle Verfahren biometrischer Kontrolle diskutiert. Dadurch wird die Herstellung der Maske in einen Dialog eingebunden, der den Austausch kritischer Informationen im Sinne der Aufklärung zum Ziel hat.

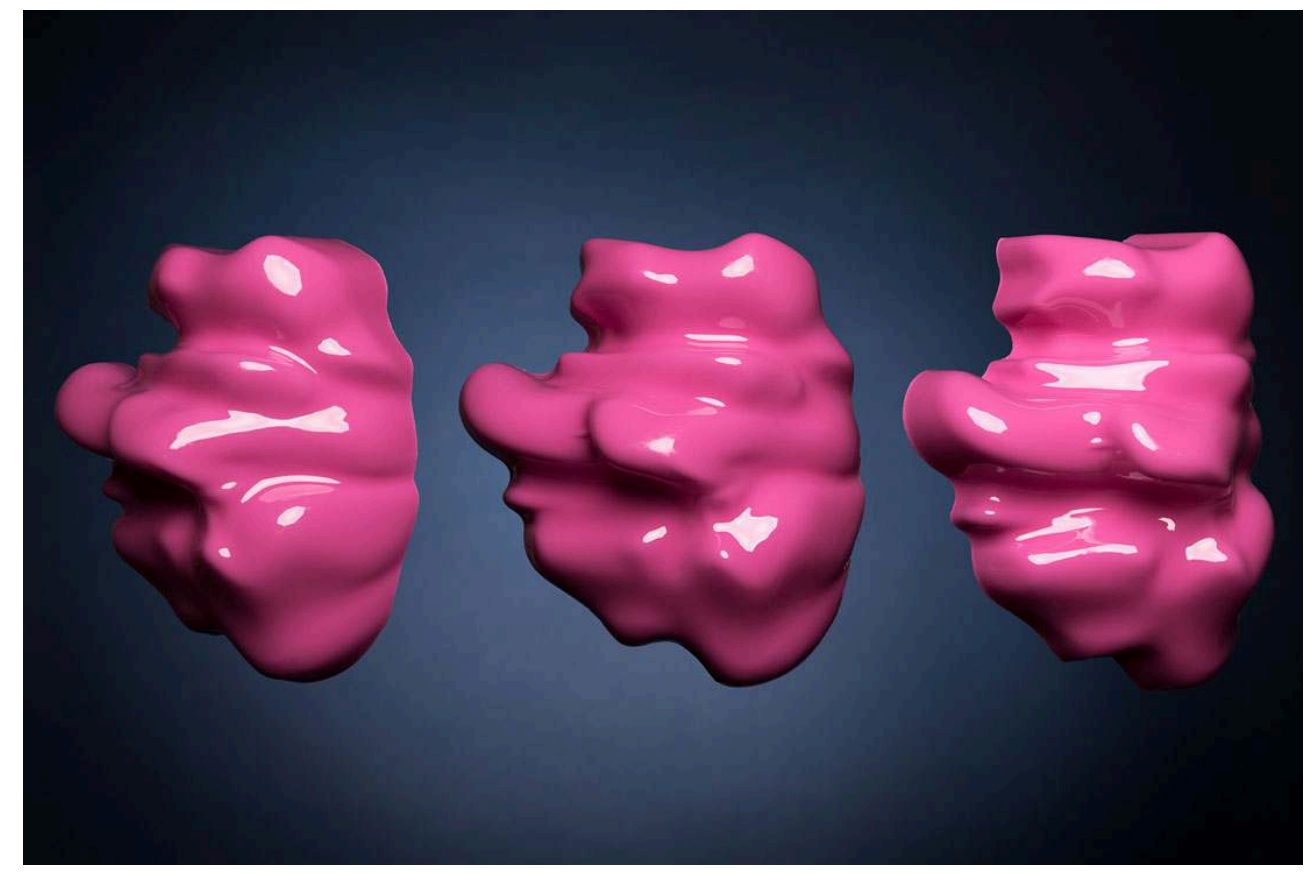

Zach Blas, Fag Face Mask, 2012

Zachs künstlerischer Ansatz richtet sich dezidiert gegen die normierenden Standardisierungsverfahren, auf denen die biometrischen Gesichtssoftwares aufbauen. Durch die einseitige und beschränkte algorithmische Software-Programmierung wird im Vermessungsverfahren jegliche Diversität, die jenseits der weißen, heterosexuellen Norm liegt, ausgespart bzw. bleibt unmessbar. Indem die Arbeit dieses reduktionistische Verfahren analysiert und visualisiert, formuliert sie einen widerständigen Protestmoment. Mit Rückbezug auf eine Studie der GenderTheoretikerin Shoshana Amielle Magnet (2011) beanstandet Blas:

Magnet explains that biometric systems, for instance, often fail to scan the hands of Asian women, eyes with cataracts, or determine age, gender, and race. Her examples indicate that the normative template for biometric functionality is white, heterosexual, and male. Taking an absolutist position, she concludes, "human bodies are not biometrifiable." a human identity cannot be captured and stabilized in a digital representation. Magnet calls for non-technical ways to discuss biometric failure and the violence it enacts (Blas 2013).

Blas' kritischer Ansatz verweist auf die seit dem 19. Jahrhundert existierende, positivistische Tradition von Mess- und Klassifizierungsverfahren des menschlichen Gesichts und Körpers, deren Anwendung zur Normierung und Diskriminierung von Personengruppen, u.a. auch auf der Kategorisierung der Hautfarbe oder sexuellen Orientierung, aufbaute. Indem Blas ein Masken-Modell nach dem Vorbild dialogischemanzipatorischer Aufklärung und die kollektive Maske der gesellschaftlich Anderen, als selbstbewusstes, kollektives Gegenbild entwickelt, führt er das Gesichtserkennungsverfahren ad absurdum. Die in seinem Workshops entwickelten Maske werden Teil einer politischen und ästhetischen Praxis mit dem Ziel, "anti- 
normative" und "anti-standardisierte" Gesichtsmasken zu entwerfen, die sich den kontrollierenden Algorithmen neoliberaler >facial detection<-Software entziehen. Seine aktionistische Herangehensweise spiegelt die Mechanismen der von Bauman/ Lyon formulierten "liquid surveillance" wieder und wehrt sie gleichermaßen ab. Gleichzeitig spielt die von Blas gewählte pinke Blasen-Ästhetik mit Baumans/Lyons Begriff der "Liquidität" und visualisiert hierdurch nicht zuletzt auch wie flüssig sich gegenwärtige Geschlechtskonzepte und soziale Zuschreibungen gestalten.

Ein weiteres Beispiel für einen subversiven Umgang mit Gesichtserkennungssoftware sind die Data Masks (2013) von Sterling Crispins (*1985, Hawai). Ähnlich wie Blas entwickelt der Künstler Gesichtsmasken, die auf der Software für biometrische Gesichtserkennung beruhen. Allerdings verfolgt Crispins dabei den umgekehrten Weg mit dem Zweck der Entvisualisierung: Seine verpixelten, unmenschlichen Masken basieren auf der algorithmischen Information menschlicher Gesichtsdatenbanken, die er mit einem "Fitness-Algorithmus", d.h. mit einem nach Vorbild des in Spionage und Privatwirtschaft verwendeten "lernfähigen" Gesichtserkennungsalgorithmus ausfindig gemacht hat, der zeigt wie Maschinen Menschen wahrnehmen. Der "frei zugängliche" Algorithmus ist unfähig, bei der Gesichtserkennung Merkmale wie Nase oder Mund zu deuten, weshalb er auf die Farbgebung der einzelnen Bildpixel reagiert. Im Anschluss verwendet Crispins sein eigens entwickeltes 3D-Software-Programm, das per Zufall auf die Farbgebung des "Fitness-Algorithmus" reagiert und sie in 3D-Modulationen verändert bis ein gesichtsähnliches Vorbild entsteht (Stöckel 2012). Die daraus resultierenden Daten verwandelt er in 3-D-Modelle und anschließend in Gips-Plastiken. Die Masken gleichen einer amorphen, ovalen Maske ohne jegliche individuelle Zuschreibungsmerkmale und verkehren damit die Funktionsweise der VisualiserungsSoftware: Das Gesicht ist durch die Visualisierung der quergeschalteten Algorithmen nicht mehr als solches erkennbar und unidentifizierbar. Darüber hinaus verfolgt Crispins das Ziel, dass die entstandenen Masken im öffentlichen Raum getragen und somit in den aktivistischen Widerstand eingebunden werden, wo sie "die Träger_innen dazu bemächtigen, den kontrollierenden Blick der Maschine zu erwidern und zu spiegeln" (Waligorski 2016). Wie Blas ist auch Crispin daran interessiert, die Mechanismen der "liquid surveillance" und facial recognition-Systeme im öffentlichen und virtuellen Raum zu unterwandern.
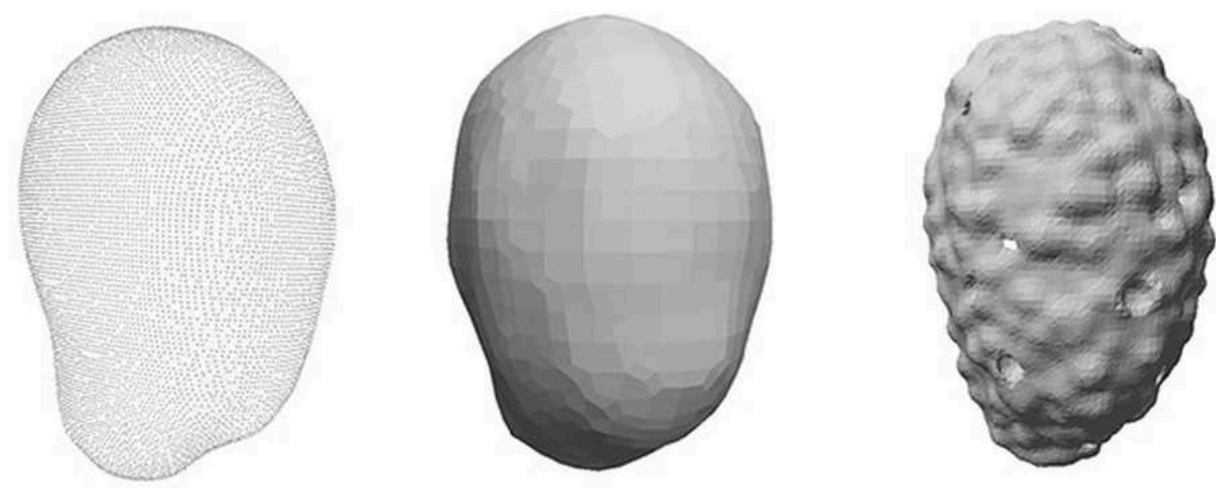

Sterling Crispin, Data Masks, 2013 
Pixelhead-Masken von Martin Backes (*1977, Deutschland) verfolgen einen ähnlichen Anspruch. Allerdings ist Backes nicht an einer 3D-Visualisierung von Gesichtssoftware interessiert, sondern entwickelt seine "Pixelheads " als flexibel einsetzbare Gesichtsmaske für den öffentlichen Raum (Backe). Selbige gleichen in ihrem Design den "Sturmkappen" aus dem Militär- und Protesthintergrund, sind jedoch nicht schwarz oder Camouflage-farben, sondern mit Pixeln bedruckt. Sie funktionieren nach Angaben des Künstlers im Sinne einer "Medien-Comouflage" und wirken der permanenten Überwachung im privaten und öffentlichen Raum entgegen: Der spezielle Pixelprint macht das Gesicht für Kameraufnahmen und Smartphone basierte Gesichtsrecherche unsichtbar, wodurch visuelle Kontrolle unmöglich wird. Im Gegensatz zu Blas und Crispins ist diese Form der Maske jedoch weitaus weniger Recherche-basiert und visualisiert den Effekt des Unsichtbar-Werdens im Sinne einer Gesichtsvermummung politischer Aktivisten.

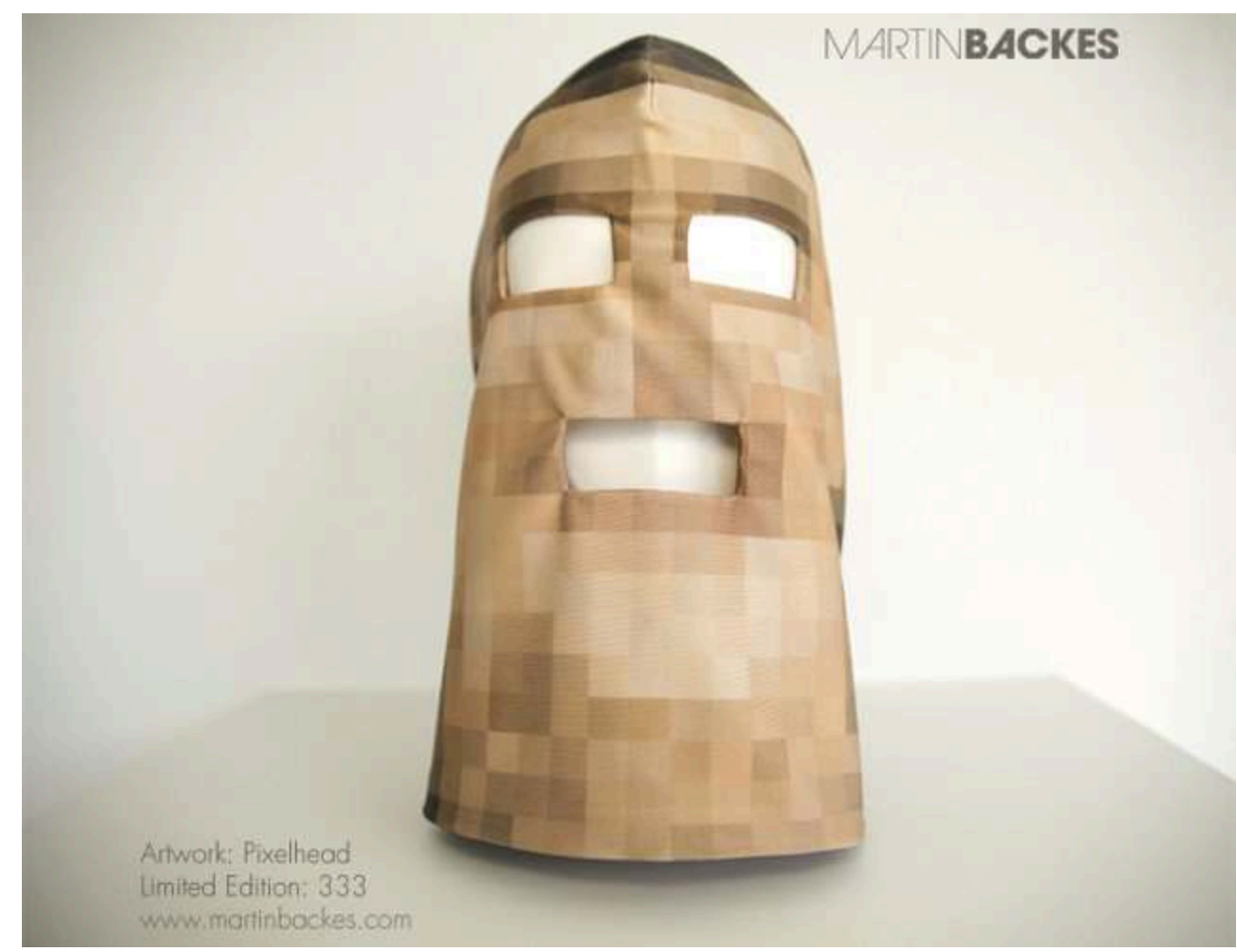

Martin Backes, Pixelhead-Masken, 2010

Wie an den genannten Beispielen ersichtlich wird, nutzen die Künstler verschiedene Masken-Formen im aktionistischen Sinne mit dem Ziel, algorithmisch basierte biometrische Gesichtskontrolle und Normierung aufzudecken oder das Gesicht für Überwachungstechnologien unkenntlich zu machen. Um dies zu erreichen entwickeln sie unterschiedliche Maskenformen, die den automatisierten Zugriff auf das Gesicht als Träger von Identitätsmerkmalen verschlüsseln, um somit eine erneute Autonomie zu erlangen. Dieser partizipative Ansatz passt zu Nicholas Mirzoeffs Konzept des "visual activism". Mirzoeff diagnostiziert einen Wandel von der mehr diskursiv angelegten "visual culture practice" der letzten 25 Jahre zu gegenwärtigen Formen des "visual activism", "that tries to imagine change" (Mirzoeff 2015: 290): Den Vertretern des "visual activism" gehe es dabei in ihren Werken, über Fragen der visuellen 
Repräsentation hinaus, vor allem um ein direktes Einwirken sowie ein aktives Engagement im Umgang mit politischen Realitäten:

In visual activist projects, there is an alternative visual vocabulary emerging. It is collective and collaborative, containing archiving, networking, researching, and mapping among other tools, all in the service of a vision of making change. [...] In 1990 , we could use visual culture to critizise and counter the way that we were depicted in art, film, and mass media. Today, we can actively use visual culture to create new self-images, new ways to see and to be seen, and new ways to see the world (Mirzoeff 2015, $292 \mathrm{f}$.).

Während die künstlerische Praxis seit den 1990er Jahren vor allem auf Fragen der visuellen Repräsentation in den Medien und in der Massenkultur reagierte und in ihren Werken bevorzugt Fragen über Geschlechterrollen und Identität aufgriff (wie u.a. etwa in dem Werk von Cindy Sherman ersichtlich), stellt Mirzoeff seit 2011 neue Strategien im Umgang mit visueller Kultur fest. Die politisch-aktivistische Motivation macht er an dem Slogan der Occupy-Proteste "They do not represent us" ("We are the other 99\%") sowie dem arabischen Frühling fest, welche Fragen der politischen Repräsentation und Teilhabe auf die Straße trugen (Mirzoeff 2016: 283 f.). Nach Mirzoeff geht es der neuen Generation von "visual activists" vor allem darum, Möglichkeiten zu finden, die Probleme unserer digital-vernetzten Gegenwart von der virtuellen in die reale Welt zu überführen und aktiv an der Veränderung politischer und gesellschaftlicher Realitäten mitzuwirken:

\begin{abstract}
Visual activism is the interaction of pixel and action to make change. Pixels are the visible result of everything produced by a computer, from words created by a word processor to all forms of image, sound, and video. Actions are things we do with those cultural forms to make changes, small or large, from direct political action to a performance - whether in everyday life or in a theatre - a conversation or a work of art. Once we have learned how to see the world, we have taken only one of the required steps. The point is to change it (Mirzoeff 2016: 293).
\end{abstract}

Dies steht im Kontrast zur früheren Generation repräsentations-kritischer Künstler_innen, welche die Gesichts-Maske im Sinne einer Kritik neuer Medien wie etwa dem Fernsehen einsetzten, wobei der virtuelle Raum und seine neuen manipulativen "Interfaces“ jedoch noch nicht in der künstlerischen Auseinandersetzung beachtetet wurden.

Das zentrale Thema des vorliegenden Essays war die Beobachtung, dass die visuelle Repräsentation des Menschen im Zuge des digitalen Wandels, neuer militärischer Bildtechnologien und der Ereignisse des 11. Septembers eine doppelte d.h. kulturphilosophische und mediale Entleerung durchläuft. Dabei interessierte mich besonders, wie es mit Mitteln der Kunst gelingen kann, den "verloren gegangenen ", individuellen Körper zu reanimieren und auf eine manipulative Bild- und Körperpolitik hinzuweisen. In einer digitalen Gesellschaft, die mit Hilfe moderner Bild- und Überwachungstechnologien und deren scheinbar unbegrenzten Möglichkeiten die Manipulation von Gesicht und Körper vorantreibt, wird das widerständige MaskenPorträt zum Symptom und gleichzeitigen Stachel. Die besprochenen Arbeiten versuchen das körperlose Oberflächengesicht aus der Flut der medialen Bilder zu befreien und es im Sinne Machos negativer Lesweise einer "facialen Gesellschaft" mit neuen Inhalten zu füllen. Durch ihre aktionistische Anlegung folgen sie der Idee eines neuen „visual actionism“ (Mirzoeff) und zielen auf einen aktiv veränderten gesellschaftlichen Diskurs im Umgang mit biometrischer Gesichtskontrolle ab, der auf eine Einbindung der Rezipienten im realen Raum setzt. 
Indem die digitalen Masken-Formen von Blas, Crispins und Backes staatliche AppAnwendungen und Software in Frage stellen, die das Gesicht vermessen, kontrollieren und überwachen wollen, üben sie Kritik an einer veränderten Körper- und Sicherheitspolitik nach dem 11. September. Der diagnostizierten Oberflächlichkeit digital produzierter, virtuell kursierender Gesichter begegnen die Künstler indem sie den verloren gegangenen, individuellen Körper sowie das Subjekt hinter der Maske wiederbeleben, um mehr Autonomie in einer permanent digital überwachten Gegenwart zu erlangen. Die künstlerischen Arbeiten entsprechen in diesem Sinne nicht zuletzt Graus Vorstellung einer kritischen "Schule des Sehens": sie vermitteln das nötige "Rüstzeug" für den Umgang mit digitalen Bildern und zeigen auf eine spielerische Weise, wie mit den neuen Herausforderungen umgegangen werden kann.

\section{BIBLIOGRAPHY}

Amelunxen, Hubertus von. 1995. „Fotografie nach der Fotografie“. In Fotografie nach der Fotografie, Ausstellungskatalog.

Backes, Martin. 2016. Homepage, Letzter Aufruf 12.9.2016. http://www.martinbackes.com/newartwork-pixelhead/

Bainbridge, Wilma A., Aditya Khosla , Antonio Torralba, and Aude Oliva. 2013. "Modifying the Memorability of Face Photographs". International Conference on Computer Vision (ICCV), 2013. Letzter Aufruf 05.05.2014. http://people.csail.mit.edu/khosla/

Bauman, Zygmunt. 2003. Flüchtige Moderne. Frankfurt am Main: Suhrkamp.

Bauman, Zygmunt \& Lyon, David. 2013. Liquid Surveillance. Cambridge: Polity Press.

Belting, Hans. 2013. Faces. Eine Geschichte Des Gesichts. München: C.H. Beck.

Benjamin, Walter. 1980. Gesammelte Schriften. Band I, Werkausgabe Band 2. Rolf Tiedemann und Hermann Schweppenhäuser (Hg.). Frankfurt am Main: Suhrkamp.

Bidgood, Jess. 2015. „Tape Found Over Portraits of Black Harvard Professors“. The New York Times. 19. November 2015. Letzter Aufruf 2.10.2018. https://www.nytimes.com/2015/11/20/us/tapefound-over-portraits-of-black-harvard-professors.html

Blas, Zach. 2013. „Escaping the Face: Biometric Facial Recognition and the Facial Weaponization Suite". Media N, new media caucus. Letzter Aufruf 12.9.2016. http://median.newmediacaucus.org/ caa-conference-edition-2013/escaping-the-face-biometric-facial-recognition-and-the-facialweaponization-suite/

Blas, Zach. 2015. "Facial Weaponization Suite”. Foam Magazine, Ausgabe \#41, "The Messenger. Image Based Activism Today", April 2015: 85- 86.

Chamayou, Grégoire. 2013. Théorie du drone. Paris: La Fabrique éditions.

Chayka, Kyle. 2014. "Face-recognition software: Is this the end of anonymity for all of us?". The Independent, 13.01.2014. Letzter Aufruf 05.05.2014. http://www.independent.co.uk/life-style/ 
gadgets-and-tech/features/facerecognition-software-is-this-the-end-of-anonymity-for-all-ofus-9278697.html\#,

Chiaverina, John. 2015. „'We didn't set out to make a piece about dehumanization, mob mentality, or violence': Alli Coates and signe Pierce Talk 'American Reflexxx'“. ARTNEWS, 5.4.2015. Letzter Aufruf 02.10.2018. http://www.artnews.com/2015/05/04/we-didnt-set-out-tomake-a-piece-about-dehumanization-mob-mentality-or-violence-alli-coates-and-signe-piercetalk-american-reflexxx/

Deleuze, Gilles \& Guattari, Felix. 1992. Tausend Plateus. Übersetzt von Gabriele Ricke und Ronald Voullié. Berlin: Merve Verlag.

De Raedt, Nele. 2015. „Defacing“. Nomoi, 03.12.2015. Letzter Aufruf 02.10.2018. https://

nomoi.hypotheses.org/261,

Flusser, Vilém. 1992. Ins Universum der technischen Bilder. Göttingen.

Grau, Oliver. 2001. Virtuelle Kunst in Geschichte und Gegenwart: Visuelle Strategien. Berlin: Dietrich Reimer Verlag.

Grau, Oliver. 1997. „In das lebendige Bild. Die Virtuelle Realität setzt der Kunst neue spielregeln“. Neue Bildende Kunst (6), 1997, 28-35. Letzter Aufruf 02.10.2018. http://www.immersence.com/ publications/1997/1997-OGrau-lebendige-bild.html

Groys, Boris. 2014. „Kunstaktivismus. Die totale Ästhetisierung der Welt als Eröffnung der politischen Aktion“. Lettre International, Nr. 106, Herbst 2014: 88-92.

Hüsch, Annette. 2007. „Künstlerische Konzeptionen am Übergang von analoger zu digitaler Fotografie“. Medien Kunst Netz. Letzter Aufruf 02.10.2018. http://www.medienkunstnetz.de/ themen/foto_byte/kuenstlerische_konzeptionen/

Kenaan, Hagi. 2013. The Ethics of Visuality: Levinas and the Contemporary Gaze. London/New York: Taurius.

Leber, Sebastian \& Rink, Tiemo. 2012. "Musik aus dem Maskenland”. Der Tagesspiegel, 9.7.2012. Letzter Aufruf 12.9.2016 http://www.tagesspiegel.de/kultur/pop/cro-und-co-musik-aus-demmaskenland/6856072.html.

Levin, Sam. 2016. „A beauty contest was judged by AI and the robots didn't like dark skin“. The Guardian, 8.9.2016. Letzter Aufruf 11.9.2016. https://www.theguardian.com/technology/2016/ sep/08/artificial-intelligence-beauty-contest-doesnt-like-black-people

Lynch, Annette \& Mitchell Strauss. 2007. Changing Fashion: A Critical Introduction to Trend Analysis and Cultural Meaning. London: Bloomsbury Academic.

Macho, Thomas. 1996. "Gesichtsverluste. Faciale Bilderfluten und postindustrieller Animismus”. In Zeitschrift für Ästhetik \& Kommunikation. Ausgabe: „Medium Gesicht: die faciale Gesellschaft”, 25-28. Berlin: Elephanten Press.

Macho, Thomas. 2011. Vorbilder. Paderborn: Wilhelm Fink.

Magnet, Shoshana Amielle. 2011. When Biometrics Fail: Gender, Race, and the Technology of Identity. Durham, NC: Duke University Press.

Mirzoeff, Nicholas. 2015. How to See the World. Gretna: Pelican Press.

Mitchel, William James. 1992. The Reconfigured Eye. Visual Truth in the Post-Photographic Era.

Cambridge/Mass. : MIT Press.

Möllers, Christoph. 2015. Die Möglichkeit der Normen. Berlin: Suhrkamp Verlag. 
Möllers, Christoph. 2016. „Das Gesicht und die Freiheit“. Süddeutsche Zeitung online. Letzter Aufruf 6.9.2016. http://www.sueddeutsche.de/kultur/debatte-das-burka-verbot-das-gesicht-und-diefreiheit-1.3147602-3

Morozov, Evgeny. 2011. „In Your Face“. In Kelly Gates, Our Biometric Future: Facial Reýcognition Technology and the Culture of Surveillance, NYU Press. Letzter Aufruf 12.9.2016. https:// evgenymorozov.tumblr.com/post/20347146711/my-piece-on-the-history-of-facial-recognition

Mozur, Paul. 2018. "Inside China's Dystopian Dreams: A.I., Shame and Lots of Cameras". The New York Times, 8.7.2018. Letzte Aufruf 28.09.2018. https://www.nytimes.com/2018/07/08/business/ china-surveillance-technology.html

Murphy, Mike. 2015. „Computers can now paint like Van Gogh and Picasso“. Quartz, 6.9.2015. Letzter Aufruf 6.9.2016. http://qz.com/495614/computers-can-now-paint-like-van-gogh-andpicasso/

Sekula, Allan. 2003. „Der Körper und das Archiv“. In Diskurse der Fotografie. Fotokritik am Ende des fotografischen Zeitalters, Band 2. (Hg.) Herta Wolf. Frankfurt am Main: Suhrkamp.

Simpson, Ian. 2017. „Statue defaced at U.S. Confederate monument protests grow“. Reuters, 18.08.2017. Letzte Aufruf 28.9.2018. https://www.reuters.com/article/us-usa-protests-statuesreaction/statue-defaced-as-u-s-confederate-monument-protests-grow-idUSKCN1AX2TK

Stöckel, Christine. 2012. „Sind das noch wir? Gesichtserkennung in der Kunst“. TAZ online, 5.12.2012. Letzter Aufruf 12.9.2016. http://www.taz.de/!5026874/

Vincent, James. 2014. "NameTag. Facial recognition app scans faces for dating profiles, criminal background”. Independent, 9.1.2014. Letzter Aufruf 15.9.2016. http://www.independent.co.uk/lifestyle/gadgets-and-tech/facial-recognition-app-scans-strangers-faces-for-dating-profilescriminal-background-9049568.html

Waligorski, Alexandra. 2016. “Anti-Gesichtserkennungsmasken”. The Nomos of Images, DIS Magazine. Letzter Aufruf 8.9.2016. https://nomoi.hypotheses.org/tag/dis-magazine

Walker, Shaun. 2016. „Face recognition app taking Russia by storm may bring end to public anonymity“. The Guardian, 17.5.2016. Letzter Aufruf 18.9.2016. https://www.theguardian.com/ technology/2016/may/17/findface-face-recognition-app-end-public-anonymity-vkontakte,

Weigel, Sigrid. 2012. "Das Gesicht als Artefakt”. Trajekte, Nr.25, 13.Jahrg., Oktober 2012: 5-13.

Weinhart, Martina. 2003. Selbstbildnis ohne Selbst. Dekonstruktion eines Genres in der zeitgenössischen Kunst. Berlin: Reimer Verlag.

Weiss, Judith Elisabeth. 2012. „Von Anti bis Meta. Neu Orientierungen der Porträtkunst.“ Kunstforum, Ausgabe: „Gesicht im Porträt / Porträt ohne Gesicht“, Band 216.

Westgeest, Helen. 2015. A Video Art Theory. A Comparative Approach. West Sussex: Wiley-Blackwell.

\section{NOTES}

1. https://prisma-ai.com/privacy.html

2. Dass die Gesichtsalgorithmen keineswegs neutral, sondern durchaus rassistisch motiviert programmiert sind, wurde zuletzt von dem Politik- und Rechtswissenschaftler Bernard Harcourt am Beispiel eines „Schönheitsalgorithmus“ belegt. Da die Software vornehmlich von weissen, männlichen Programmierern angelegt wird, entstehen stereotype Vorstellungen von Schönheit, 
die sich durchaus mit den Anfängen der Rassenfotografie des 19./20. Jahrhunderts vergleichen lassen. Vgl. Levin 2016.

3. Der Einfluss des CCTVs auf die künstlerische Produktion ist von grosser Bedeutung und schlägt sich u.a. im Werk von Bruce Nauman (Live/Taped Video Corridor, 1969-70) oder Nam Jun Paik (TV Buddah, 1974) nieder.

4. American Reflexxx basiert auf einer Performance, die 2015 in Zusammenarbeit mit der Filmemacherin Alli Coates entstand und dokumentiert filmisch den Spiessrutenlauf einer maskierten Unbekannten durch das Stadtzentrum in Mrytle Beach, South Carolina. Ihr Gesicht ist durch eine spiegelnde Maske maskiert, der sperrlich bedeckte Körper zeigt transsexuelle Geschlechtsmerkmale. Im Verlauf des 15min. Zusammenschnitt des Films, der u.a. im Palais de Tokyo zu sehen war und auch auf Youtube zugänglich ist, wird die Protagonisten von verschiedensten Menschen auf der Strasse beleidigt, angespuckt und schliesslich von einem Mob brutal auf den Boden gestossen. Vgl. Chiaverina 2015.

\section{ABSTRACTS}

Das Gesichts- und Körperbild der Gegenwart unterliegt einem neuen Regime der Transparenz: Digitale Anwendungen wie Facebook, Biometrik und Gesichtserkennungstechnologien definieren Gesichtlichkeit und Sichtbarkeit vor allem durch neue Parameter der Überwachungskultur. Als Reaktion auf diese Entwicklung, produzieren viele zeitgenössische Künstlerinnen und Künstler Bilder des menschlichen Gesichtes, die die konventionelle Simplifizierung algorithmisch definierter Abbilder durch subversive Akte der Maskerade unterwandern. Dieser Artikel untersucht die unterschiedlichen Konzeptionen von Gesichtlichkeit, Sichtbarkeit und Körperpolitik im Zeitalter des Digitalen in den künstlerischen Arbeiten von Zach Blas, Sterling Crispins und Martin Backes. Ihre Werken begegnen dem Verlust der Autonomie, indem sie aktivistische Ansätze entwickeln, die die Strategien der Überwacher gegen diese selbst lenken.

The body- and face-image of the present is determined by a new regime of transparency. Digital tools such as Facebook, biometrics and techniques of face recognition are redefining the visibility of bodies and faces following the new parameters of the surveillance culture. In reaction to these developments, several contemporary artists are producing images of the human face that infiltrate, by means of subversive actions of masking, the conventional simplification as it is normally defined by algorithms. This article investigates the different conceptions of face, appearance and body politics at the time of the digital in work of artists such as Zach Blach, Sterling Crispins and Martin Backes. Their work confronts the lost of autonomy and develops an activist approach that subverts the surveillance strategy.

L'image actuelle $d u$ visage et $d u$ corps présuppose un nouveau régime de transparence. C'est surtout selon les nouveaux paramètres de la culture de surveillance que des outils numériques tels que Facebook, la biométrique et les technologies de reconnaissance faciale redéfinissent les modes d'apparaître du corps et du visage. Réagissant à ce développement, de nombreux artistes contemporains produisent de multiples images du visage humain qui infiltrent, par des actes subversifs de masquage, la simplification conventionnelle du portrait tel qu'il est habituellement défini par les algorithmes. Cet article étudie les différentes conceptions du visage, de l'apparence et de la politique des corps à l'époque du numérique dans des œuvres d'artistes comme Zach 
Blach, Sterling Crispins et Martin Backes. Leurs œuvres se confrontent à la perte de l'autonomie et, à partir de là, développent une approche activiste qui retourne les stratégies de surveillance contre elles-mêmes.

La imagen actual del rostro y del cuerpo presupone un nuevo régimen de transparencia. Herramientas digitales como Facebook, la biometría y las tecnologías de reconocimiento facial redefinen los modos de aparecer del cuerpo y del rostro según los nuevos parámetros de la cultura de la vigilancia. Como respuesta a este desarrollo, muchos artistas contemporáneos producen imágenes del rostro humano que, a través de actos subversivos de enmascaramiento, se infiltran en la simplificación convencional de las imágenes tal como son definidas mediante algoritmos. Este artículo estudia las diferentes concepciones del rostro, de la visibilidad y de la política del cuerpo en la era digital en las obras artísticas de Zach Blas, Sterling Crispins y Martin Backes. Sus trabajos se confrontan a la pérdida de autonomía desarrollando enfoques activistas que subvierten las estrategias de vigilancia contra sí mismas.

\section{INDEX}

Mots-clés: Art et activiste, culture du visage numérique, transparence à l'âge de la postphotographie, surveillance politique, éthique de l'algorithme

Schlüsselwörter: Kunst und Aktivismus, Digitale Gesichtskultur, Transparenz im Zeitalter der Post-Fotographie, Politik der Überwachung, Ethik der Algorithmen

Keywords: Art and activism, digital face culture, transparency in the age of post-photography, surveillance politics, the ethics of algorithms

Palabras claves: Arte y activismo, cultura digital facial, transparencia en la era de la postfotografía, vigilancia política, ética de los algoritmos

\section{AUTHOR}

\section{JANA JOHANNA HAECKEL}

Ph.D. Senior researcher at Lieven Gevaert Research Centre For Photography jana.haeckel@gmail.com 